\title{
An assessment of preoperative evaluation modalities in predicting muscle biopsy diagnostic yield and therapeutic impact
}

\author{
Tenny $\mathrm{SO}^{1 *}$, Schmidt KP${ }^{1}$, Marmie $\mathrm{BR}^{2}$ and Follett $\mathrm{KA}^{\mathbf{1}}$ \\ ${ }^{1}$ Division of Neurosurgery, University of Nebraska Medical Center, USA \\ ${ }^{2}$ School of Medicine, University of Nebraska Medical Center, USA
}

\begin{abstract}
Background/Objectives: Muscle biopsies are requested for the workup of myopathy when less invasive workup is inconclusive. We set forth to build a multivariate model of preoperative testing to increase the odds of diagnostic and therapeutic yield of a muslce biopsy.

Methods: A 5-year retrospective chart review of 106 patients who underwent muscle biopsy was performed to evaluate preoperative workup including: magnetic resonance imaging, electromyography, muscle strength testing and laboratory evaluation including creatine kinase level, lactate level, urine myoglobin, acetylcholinesterase antibody testing, genetic testing or use of steroids in the preoperative period using Student's two-tailed t-test, two-tailed Fisher's exact test and multivariate regression model.

Results: We found an overall biopsy diagnostic pathologic yield of $47 \%$ and therapeutic yield of $88 \%$. None of the variables reached a level of significance of p-value $<0.05$ in univariate testing. In the multivariate model preoperative use of steroids and creatine kinase were the closest predictors of diagnostic and therapeutic yield but failed to reach statistical significance.

Conclusions: The therapeutic impact of muscle biopsies appears to be much greater than the diagnostic pathologic yield and the therapeutic impact larger than previously reported when rigorously defined. We were unable to create a statistically significant preoperative predictive model for muscle biopsy diagnostic and therapeutic yield.
\end{abstract}

\section{Introduction}

Muscle biopsies have a crucial role in the evaluation of neuromuscular conditions, especially myopathy. The evaluation of myopathic processes can be lengthy and many times non-definitive due to the variable, non-focal signs and symptoms on initial presentation and evaluation. Undergoing specific physical examination $[1,2]$, laboratory evaluation, electrodiagnostic evaluation [2-4], and imaging [5] may lead to a diagnosis without need for muscle biopsy. When workup is nondefinitive a muscle biopsy may be pursued. The current literature lacks a consistent model to define preoperative factors which increase diagnostic or therapeutic yield of muscle biopsies.

Muscle biopsies have inherent surgical risks [6] while providing variable diagnostic and therapeutic outcomes [7-12] and are thus no pancea. We set out to provide an analysis of frequently encountered myopathy evaluation techniques and the relationship to diagnostic biopsy result or therapeutic impact to maximize muscle biopsy utility.

\section{Materials and methods}

We performed a single institution chart review for all muscle biopsies from January 1, 2012 through July 1, 2016. Inclusion criteria for the chart review were: age 19 years or older at time of the muscle biopsy (the age of majority in the state of the study) and charted for Current Procedural Terminology (CPT(R) AMA, 2016) code 20200 (superficial muscle biopsy) or 20205 (deep muscle biopsy). Each chart identified to match the search criteria was then abstracted for: age at biopsy, gender, muscle biopsied, pathology diagnosis from pathology report, therapeutic implications of the muscle biopsy, physical exam documentation prior to biopsy, and pre-operative workup including presence or absence of body magnetic resonance imaging (MRI) to identify muscle site for biopsy, electromyography (EMG), creatine kinase (CK) level, lactate level, urine myoglobin level, acetylcholinesterase antibody testing, use of steroids in the one-month immediate pre-operative period and any pre-operative genetic testing relating to the workup of myopathy. Pathology results were captured as text an element. Pre-operative physical exam, MRI for selection of biopsy location, EMG, laboratory testing and use of steroids was noted as either present or absent without regards to the specific levels of the tests.

The endpoints examined were used to build a multivariate model based on pre-operative workup to determine what aspects of the preoperative workup are most likely to lead to a higher definitive pathologic diagnostic yield or therapeutic benefit of the muscle

Correspondence to: Tenny SO, Department of Surgery, Division of Neurosurgery, 982035 Nebraska Medical Center Omaha, NE 68198-2035, USA, Tel: 402-5599605; E-mail. steven.tenny@unmc.edu

Key words: diagnostic yield, muscle biopsy, preoperative workup, therapeutic impact

Received: January 22, 2018; Accepted: February 20, 2018; Published: February 23,2018 
biopsy. Definitive pathologic diagnosis was defined as a pathology report which provided a conclusive result. Definitive pathologic results identified included: amyloid from multiple myeloma, Becker muscular dystrophy, consistent with myotonic dystrophy 2, dermatomyositis, immune myopathy, inclusion body myositis, inflammation consistent with polymyositis, inflammatory myositis, invasive large B-cell lymphoma, myeloid sarcoma, mitochondrial myopathy, necrotizing myelopathy, and polymyositis.

Non-conclusive pathology reports were unable to provide a definitive pathologic diagnosis. Pathologic results that were nondefinitive included: chronic myopathic changes, end-stage muscle atrophy, fatty replacement, mild non-specific atrophy, myopathy not otherwise specified, negative, no diagnostic alterations, non-diagnostic, possible myopathy, possible neurogenic atrophy, rare macrophages, scant inflammation, suspicious for microvasculitis, type II atrophy or if the pathology report included a list of differential diagnoses.

Therapeutic implication of the biopsy was determined by looking at post-operative notes from the referring service which referred to the pathology report. Documentation which stated the referring service was either planning on offering new treatment or stopping or continuing or modifying current treatment based on the pathology results in context of the pre-operative workup were considered as positive for therapeutic implication. If no mention of the pathology report could be identified or the documentation did not specify changes in treatment course based on the pathology report then the muscle biopsy was considered to not have therapeutic implications.

All pathology was scored independently as diagnostic or nondiagnostic as well as therapeutic or non-therapeutic. In any cases where discrepancies arose the decision was resolved with group consensus (KPS and SOT) and documented for future reference and to help ensure continued consistency.

Continuous variables were analyzed using an independent sample two-tailed t-test with a predefined alpha of 0.05 . Categorical variables were analyzed using a two-tailed Fisher's exact test with a predefined alpha of 0.05 . We examined all pre-operative variables with the outcome variable of definitive pathologic diagnosis and therapeutic implication of the biopsy. In building our multivariate regression model we only tested for variables with a univariate $\mathrm{p}$-value $<0.1$. We pre-defined inclusion of variables in a multivariate regression model as significant if they had a p-value $<0.05$. Data was analyzed using SAS version 9.4 (SAS Institute Inc., Cary, NC) by the statistician.

Prior to identifying charts of interest or commencing chart abstraction the project received approval from the home institutions Internal Review Board for protection of human subjects (IRB 502-16-EX).

\section{Results}

A total of 106 muscle biopsies were identified. The ages ranged from 21 to 86 years with a mean of 57.4 years and standard deviation of 16.9 years. Fifty-five percent of biopsies were from males and $45 \%$ from females. Muscles biopsied were 34 vastus lateralis, 27 quadriceps (not otherwise specified), 18 deltoid, 9 biceps, 4 rectus femoris, 3 each of vastus medialis, paraspinous and triceps, 2 gluteus, and one each of trapezius, hamstring, and gastrocnemius. Neurology requested $68 \%$ of the biopsies, rheumatology $29 \%$ of the biopsies and family medicine, oncology and gastroenterology $1 \%$ each of the biopsies.

Forty-seven percent of biopsies yielded definitive pathology and $53 \%$ of biopsies had non-definitive pathology. The biopsy provided therapeutic benefit in $88 \%$ of biopsies and no therapeutic benefit in $12 \%$ of biopsies.

Table 1 shows the univariate analysis of variables relating to definitive pathologic diagnosis. Of note, the muscle biopsied variable had too many categories with small values to perform meaningful or valid statistical testing. The muscle biopsied is thus assumed to be not statistically significant. The p-values for the univariate analysis ranged

Table 1. Fisher exact test analysis on predictive value of various sample characteristics on finding definitive pathology.

\begin{tabular}{|c|c|c|c|c|c|}
\hline \multicolumn{6}{|c|}{ Sample Criteria predictive value on Definitive Pathological findings } \\
\hline \multirow[t]{2}{*}{$\begin{array}{c}\text { Patient } \\
\text { Characteristics }\end{array}$} & No & & Yes & & \\
\hline & Count & Percent & Count & Percent & p-value \\
\hline Sex & 29 & $53.70 \%$ & 29 & $55.70 \%$ & \\
\hline \multicolumn{6}{|l|}{ Male } \\
\hline Female & 25 & $46.20 \%$ & 23 & $44.20 \%$ & 0.85 \\
\hline Biopsy & 14 & $25.90 \%$ & 20 & $38.40 \%$ & \\
\hline \multicolumn{6}{|l|}{ Vast lateralis } \\
\hline Quad & 16 & $29.60 \%$ & 11 & $21.10 \%$ & \\
\hline Trap & . & . & 1 & $1.90 \%$ & \\
\hline Deltoid & 11 & $20.30 \%$ & 7 & $13.40 \%$ & \\
\hline Hamstring & . & . & 1 & $1.90 \%$ & \\
\hline Gluteal & 2 & $3.70 \%$ & . & . & \\
\hline Vast med & 1 & $1.80 \%$ & 2 & $3.80 \%$ & \\
\hline Bicep & 5 & $9.20 \%$ & 4 & $7.60 \%$ & \\
\hline Paraspinalis & 1 & $1.80 \%$ & 2 & $3.80 \%$ & \\
\hline Rectus femoris & 2 & $3.70 \%$ & 2 & $3.80 \%$ & \\
\hline Triceps & 2 & $3.70 \%$ & 1 & $1.90 \%$ & \\
\hline Gastrocnemius & . & . & 1 & $1.90 \%$ & $*$ \\
\hline PE weakness & 5 & $9.20 \%$ & 2 & $3.80 \%$ & \\
\hline \multicolumn{6}{|l|}{ No } \\
\hline Yes & 49 & $90.70 \%$ & 49 & $94.20 \%$ & \\
\hline 99 & . & . & 1 & $1.90 \%$ & 0.44 \\
\hline$C K$ & 3 & $5.50 \%$ & 2 & $3.80 \%$ & \\
\hline \multicolumn{6}{|l|}{ No } \\
\hline Yes & 51 & $94.40 \%$ & 50 & $96.10 \%$ & 1 \\
\hline Pyruvate & 54 & $100.00 \%$ & 52 & $100.00 \%$ & \\
\hline \multicolumn{6}{|l|}{ No } \\
\hline Lactate & 44 & $81.40 \%$ & 38 & $73.00 \%$ & \\
\hline \multicolumn{6}{|l|}{ No } \\
\hline Yes & 10 & $18.50 \%$ & 14 & $26.90 \%$ & 0.36 \\
\hline Urine myoglobin & 53 & $98.10 \%$ & 49 & $94.20 \%$ & \\
\hline \multicolumn{6}{|l|}{ No } \\
\hline Yes & 1 & $1.80 \%$ & 3 & $5.70 \%$ & 0.36 \\
\hline Acetylcholine abx & 30 & $55.50 \%$ & 30 & $57.60 \%$ & \\
\hline \multicolumn{6}{|l|}{ No } \\
\hline Yes & 24 & $44.40 \%$ & 22 & $42.30 \%$ & 0.85 \\
\hline$M R I$ & 40 & $74.00 \%$ & 34 & $65.30 \%$ & \\
\hline \multicolumn{6}{|l|}{ No } \\
\hline Yes & 14 & $25.90 \%$ & 18 & $34.60 \%$ & 0.4 \\
\hline$E M G$ & 12 & $22.20 \%$ & 15 & $28.80 \%$ & \\
\hline \multicolumn{6}{|l|}{ No } \\
\hline Yes & 42 & $77.70 \%$ & 37 & $71.10 \%$ & 0.51 \\
\hline Steroids & 41 & $75.90 \%$ & 36 & $69.20 \%$ & \\
\hline \multicolumn{6}{|l|}{ No } \\
\hline Yes & 13 & $24.00 \%$ & 16 & $30.70 \%$ & 0.52 \\
\hline Genetic testing & 51 & $94.40 \%$ & 50 & $96.10 \%$ & \\
\hline \multicolumn{6}{|l|}{ No } \\
\hline Yes & 3 & $5.50 \%$ & 2 & $3.80 \%$ & 1 \\
\hline
\end{tabular}

"For biopsy, there were to many categories with small expected cell counts to calculate a p-value using Fisher's exact test. 
from 0.36 to 1.00 and none met the 0.1 threshold to be included in a multivariate model.

Table 2 shows the univariate analysis of variables relating to the therapeutic benefit of the biopsy. Again noted is the paucity of samples for some of the muscles biopsied thus no meaningful or valid statistical testing could be performed for muscle biopsied versus therapeutic benefit of the muscle biopsy. Muscle biopsied is thus assumed to not be

Table 2. Fisher exact test analysis on predictive value of various sample characteristics on treatment alterations

\begin{tabular}{|c|c|c|c|c|c|}
\hline \multicolumn{6}{|c|}{ Sample Criteria predictive value on Treatment Alterations } \\
\hline $\begin{array}{c}\text { Patient } \\
\text { Characteristics }\end{array}$ & No & & Yes & & \\
\hline & Count & Percent & Count & Percent & p-value \\
\hline Sex & 5 & $38.40 \%$ & 53 & $56.90 \%$ & \\
\hline \multicolumn{6}{|l|}{ Male } \\
\hline Female & 8 & $61.50 \%$ & 40 & $43.00 \%$ & 0.24 \\
\hline Biopsy & 3 & $23.00 \%$ & 31 & $33.30 \%$ & \\
\hline \multicolumn{6}{|l|}{ Vast lateralis } \\
\hline Quad & 5 & $38.40 \%$ & 22 & $23.60 \%$ & \\
\hline Trap & . & . & 1 & $1.00 \%$ & \\
\hline Deltoid & 4 & $30.70 \%$ & 14 & $15.00 \%$ & \\
\hline Hamstring & . & . & 1 & $1.00 \%$ & \\
\hline Gluteal & 1 & $7.60 \%$ & 1 & 0.01 & \\
\hline Vast med & . & . & 3 & $3.20 \%$ & \\
\hline Bicep & . & . & 9 & $9.60 \%$ & \\
\hline Paraspinalis & . & . & 3 & $3.20 \%$ & \\
\hline Rectus femoris & . & . & 4 & $4.30 \%$ & \\
\hline Triceps & . & . & 3 & $3.20 \%$ & \\
\hline Gastrocnemius & . & . & 1 & $1.00 \%$ & $*$ \\
\hline PE weakness & 1 & $7.60 \%$ & 6 & $6.40 \%$ & \\
\hline \multicolumn{6}{|l|}{ No } \\
\hline Yes & 12 & $92.30 \%$ & 86 & $92.40 \%$ & \\
\hline 99 & . & . & 1 & $1.00 \%$ & 1 \\
\hline$C K$ & 2 & $15.30 \%$ & 3 & $3.20 \%$ & \\
\hline \multicolumn{6}{|l|}{ No } \\
\hline Yes & 11 & $84.60 \%$ & 90 & $96.70 \%$ & 0.11 \\
\hline Pyruvate & 13 & $100.00 \%$ & 93 & $100.00 \%$ & \\
\hline \multicolumn{6}{|l|}{ No } \\
\hline Lactate & 9 & $69.20 \%$ & 73 & $78.40 \%$ & \\
\hline \multicolumn{6}{|l|}{ No } \\
\hline Yes & 4 & $30.70 \%$ & 20 & $21.50 \%$ & 0.49 \\
\hline Urine myoglobin & 13 & $100.00 \%$ & 89 & $95.60 \%$ & \\
\hline \multicolumn{6}{|l|}{ No } \\
\hline Yes & . & . & 4 & $4.30 \%$ & 1 \\
\hline Acetylcholine abx & 7 & $53.80 \%$ & 53 & $56.90 \%$ & \\
\hline \multicolumn{6}{|l|}{ No } \\
\hline Yes & 6 & $46.10 \%$ & 40 & $43.00 \%$ & 1 \\
\hline MRI & 9 & $69.20 \%$ & 65 & $69.80 \%$ & \\
\hline \multicolumn{6}{|l|}{ No } \\
\hline Yes & 4 & $30.70 \%$ & 28 & $30.10 \%$ & 1 \\
\hline EMG & 4 & $30.70 \%$ & 23 & $24.70 \%$ & \\
\hline \multicolumn{6}{|l|}{ No } \\
\hline Yes & 9 & $69.20 \%$ & 70 & $75.20 \%$ & 0.74 \\
\hline Steroids & 12 & $92.30 \%$ & 65 & $69.80 \%$ & \\
\hline \multicolumn{6}{|l|}{ No } \\
\hline Yes & 1 & $7.60 \%$ & 28 & $30.10 \%$ & 0.11 \\
\hline Genetic testing & 12 & $92.30 \%$ & 89 & $95.60 \%$ & \\
\hline \multicolumn{6}{|l|}{ No } \\
\hline Yes & 1 & $7.60 \%$ & 4 & $4.30 \%$ & 0.49 \\
\hline
\end{tabular}

"For biopsy, there were to many categories with small expected cell counts to calculate a p-value using Fisher's exact test. statistically significant. The p-values for the univariate analysis ranged from 0.11 to 1.00 and none met the 0.1 threshold to be included in a multivariate model.

\section{Discussion}

Our study's muscle biopsy therapeutic impact findings are notably higher than previously documented in the literature with $88 \%$ of the biopsies we reviewed demonstrating therapeutic relevance. The muscle biopsy diagnostic yield finding of $47 \%$, however, remains consistent with previously reported findings of $13-60 \%[1,2,4,5,9,10,13,14]$. Similar to the diagnostic yield findings, our evaluation of preoperative factors and the effect on definitive muscle biopsy pathology and therapeutic implications did not demonstrate significant findings in what we believe is the most inclusive study of muscle biopsy preoperative evaluation techniques to date, albeit not the largest.

Previous changes in treatment based on muscle biopsy are reported as $8.7 \%$ [9], 19\% [15], and 33\% [10] compared to our therapeutic yield of $88 \%$. We believe there are multiple factors for this discrepancy. Firstly, the previous studies only identified changes in treatment. $[9,10,15]$. We believe therapeutically important pathologic findings are much more than those findings that justify changes in treatment. We identified multiple instances in our data where non-diagnostic pathologic reports provided additional data which allowed the treatment team to justify continuation of the current treatment. A second factor which may contribute to the differences is the time frame of the therapeutic yield. Many of the notes which referred to the biopsy result occurred weeks to two months after biopsy. The previous literature does not specify over what time frame they looked for changes in treatment nor how they identified treatment changes. This makes it more difficult to compare our results to the previous literature.

In the aforementioned studies Lai et al. [10] examined a more limited number of factors than the current study affecting therapeutic yield of muscle biopsy and found that a normal CK level and negative family history for myopathy were more likely to lead to therapeutic implications. Shaibani et al. [2] noted the combination of hyper CKemia, proximal weakness and myopathic findings on EMG predicted increased diagnostic yield of muscle biopsies but did not correlate this to therapeutic implications of the biopsy.

Our inability to associate $\mathrm{CK}$ with diagnostic or therapeutic yield noted in previous literature $[2,10]$ may be multifactorial. Our smaller sample size than the 258 [10] or 698 [2] biopsies of the other studies limits our ability to find subtle effects. There is also controversy as to what CK level is an appropriate threshold. Shaibani et al. [2] compared mean CK levels of their normal muscle biopsies (1174 IU/L) to the abnormal muscle biopsies mean CK level (2508 IU/L). This contrasts to Lai et al. [10] who used a normal CK reference range (24-195 IU/L) compared to any CK level outside the normal range to find statistical significance for diagnostic muscle biopsies. This makes it more difficult to compare between the two studies. Both studies, however, agree the larger the CK level the more likely there is to be abnormal findings on the muscle biopsy. Interestingly, neither study analyzes the results if no CK level is obtained versus obtaining a CK level which our study tried to answer as 5 of 106 muscle biopsies in our study were performed on patients without a documented or mentioned CK level preoperatively.

Another potential factor contributing the reported wide range of diagnostic muscle biopsy yields is the numerous and varying preoperative evaluation techniques between referring physicians and the interpretation of these as this is what ultimately lead to biopsy. 
Several publications have evaluated specific preoperative evaluation modalities $[1-4,10]$ with variable results. While our study did not have significant findings in regard to preoperative muscle biopsy evaluation modalities predicting resultant diagnostic pathologic or therapeutically important biopsies our findings support previous literatures' variable results as our evaluation was more inclusive of preoperative evaluation than previous studies to the best of our knowledge.

Although we failed to find any statistically significant variables in our univariate model, we performed a post-hoc analysis to examine the two most likely suspects to affect diagnostic and therapeutic yield: steroid use and CK testing. In multivariate model analysis neither of these variables reached a statistical level of significance. Steroid use in the immediate preoperative period had a 1.394 odds ratio $(95 \% \mathrm{CI}$ 0.590 - 3.289) of affecting diagnostic yield and 5.121 (95\% CI 0.624 42.002) of affecting therapeutic yield. Likewise CK testing had a 1.426 (95\% CI 0.229 - 9.006) odds of affecting diagnostic yield and 5.361 (95\% CI 0.756 - 38.032) odds of affecting therapeutic yield for a muscle biopsy.

There are some important limitations to note with our study. Our study population was adult patients referred to a tertiary academic center with neuropathologic review of slides which may not be generalizable to all situations. Additionally our definitions of diagnostic and therapeutic yield may differ from previous studies. We have tried to define our criteria for diagnostic and therapeutic yield sufficently to allow for future comparisons. Our study may be underpowered to detect subtle differences in results and build a more nuanced predictive model.

\section{Conclusion}

We found a higher muscle biopsy therapeutic yield of $88 \%$ compared to previously reported literature. In our analysis of preoperative factors we were unable to identify a statistically significant model for prediction of diagnostic or therapeutic yield of muscle biopsies. Our study bolsters prior literature on the need for further investigation to examine preoperative factors which affect the diagnostic and therapeutic yield of muscle biopsies.

\section{Acknowledgements}

We would like to acknowledge our statistician Elizabeth Lyden who performed the data analysis and medical student Frank Mezzacappa who assisted with chart abstraction.

\section{Conflict of interest}

None of the authors have any financial conflict of interest to report related to this research.

\section{Financial support}

No funding was received for this project

\section{References}

1. Miller S, Shevell M, Silver K, Kramer M (1998) The diagnostic yield of the nervemuscle skin biopsy in paediatric neurology practice. Pediatr Rehabil 2: 95-100. [Crossref]

2. Shaibani A, Jabari D, Jabbour M, Arif C, Lee M, et al. (2015) Diagnostic outcome of muscle biopsy. Muscle Nerve 51: 662-668. [Crossref]

3. Buchthal F, Kamieniecka Z (1982) The diagnostic yield of quantified electromyography and qualified muscle biopsy in neuromuscular disorders. Muscle Nerve 5: 265-280. [Crossref]

4. Hausmanowa-Petrusewicz I, Kaminska AM (1984) Diagnositic yield of quantitative electromyography and muscle biopsy in myopathic diseases. Neuropat Pol 22: 307 334

5. Lovitt S, Marden FA, Gundogdu B, Ostrowski ML (2004) MRI in myopathy. Neurol Clin 22: 509-538, v. [Crossref]

6. Hart MG, Santarius T, Trivedi RA (2013) Muscle and nerve biopsy for the neurosurgical trainee. Br J Neurosurg 27: 727-734. [Crossref]

7. Chen DJ, Prayson RA (2014) Evaluation of simultaneous muscle and nerve biopsies for the diagnosis of neuromuscular diseases. Ann Diagn Pathol 18: 282-285. [Crossref]

8. Claussen GC, Thomas TD, Goyne C, Vázquez LG, Oh SJ (2000) Diagnostic value of nerve and muscle biopsy in suspected vasculitis cases. J Clin Neuromuscul Dis 1: 117-123. [Crossref]

9. Jamshidi R, Harrison MR, Lee H, Nobuhara KK, Farmer DL (2008) Indication for pediatric muscle biopsy determines usefulness. J Pediatr Surg 43: 2199-2201. [Crossref]

10. Lai CH, Melli G, Chang YJ, Skolasky RL, Corse AM, et al. (2010) Open muscle biopsy in suspected myopathy: diagnositc yield and clinical utility. Eur J Neurol 17: 136-142. [Crossref]

11. Magistris MR, Kohler A, Pizzolato G, Morris MA, Baroffio A, et al. (1998) Needle muscle biopsy in the investigation of neuromuscular disorders. Muscle Nerve 21: 194200. [Crossref]

12. Skram MK, Gulati S, Larsson E, Lindal S, Torp SH (2009) Muscle biopsies in children - an evaluation of histopathology and clinical value during a 5-year period. Ups J Med Sci 114: 41-45. [Crossref]

13. Prayson RA (2006) Diagnostic yield associated with multiple simultaneous skeletal muscle biopsies. Am J Clin Pathol 126: 843-848. [Crossref]

14. Vladutiu GD (2002) Laboratory diagnosis of metabolic myopathies. Muscle Nerve 25 649-663. [Crossref]

15. Reynolds EM, Thompson IM, Nigro MA, Kupsky WJ, Klein MD (1999) Muscle and nerve biopsy in the evaluation of neuromuscular disorders: the surgeon's perspective. $J$ Ped Surg 34: 558-590. [Crossref]

Copyright: (C2018 Tenny SO. This is an open-access article distributed under the terms of the Creative Commons Attribution License, which permits unrestricted use, distribution, and reproduction in any medium, provided the original author and source are credited. 